\title{
Nonperturbative Infrared Finiteness in a Superrenormalizable Scalar Quantum Field Theory
}

\author{
Guido Cossu®, ${ }^{1,2}$ Luigi Del Debbio $\odot,{ }^{2}$ Andreas Jüttner $\odot,{ }^{3,4, *}$ Ben Kitching-Morley $\odot,{ }^{3,5,4}$ Joseph K. L. Lee๑, ${ }^{2}$ \\ Antonin Portelli®, ${ }^{2}$ Henrique Bergallo Rocha๑, ${ }^{2}$ and Kostas Skenderis $\odot^{5,4}$ \\ (LatCos Collaboration) \\ ${ }^{1}$ Braid Technologies, Shibuya 2-24-12, Tokyo, Japan
${ }^{2}$ Higgs Centre for Theoretical Physics, School of Physics and Astronomy, The University of Edinburgh, \\ Edinburgh EH9 3FD, United Kingdom \\ ${ }^{3}$ School of Physics and Astronomy, University of Southampton, Southampton SO17 1BJ, United Kingdom \\ ${ }^{4}$ STAG Research Center, University of Southampton, Highfield, Southampton SO17 1BJ, United Kingdom \\ ${ }^{5}$ Mathematical Sciences, University of Southampton, Highfield, Southampton SO17 1BJ, United Kingdom
}

(Received 11 December 2020; accepted 29 April 2021; published 4 June 2021)

\begin{abstract}
We present a study of the IR behavior of a three-dimensional superrenormalizable quantum field theory consisting of a scalar field in the adjoint of $S U(N)$ with a $\varphi^{4}$ interaction. A bare mass is required for the theory to be massless at the quantum level. In perturbation theory, the critical mass is ambiguous due to IR divergences, and we indeed find that at two loops in lattice perturbation theory the critical mass diverges logarithmically. It was conjectured long ago in [R. Jackiw et al., Phys. Rev. D 23, 2291 (1981), T. Appelquist et al., Phys. Rev. D 23, 2305 (1981)] that superrenormalizable theories are nonperturbatively IR finite, with the coupling constant playing the role of an IR regulator. Using a combination of Markov Chain Monte Carlo simulations of the lattice-regularized theory, frequentist and Bayesian data analysis, and considerations of a corresponding effective theory, we gather evidence that this is indeed the case.
\end{abstract}

DOI: 10.1103/PhysRevLett.126.221601

Introduction.-Massless superrenormalizable quantum field theories suffer from severe IR divergences in perturbation theory: the same power counting argument that implies good UV behavior also implies bad IR behavior. Explicit perturbative computations (with an IR regulator) lead to IR logarithms that make the perturbative results ambiguous. The fate of the IR singularities was discussed in [1,2], where it was argued that such theories are nonperturbatively IR finite. In the examples analyzed in [1,2], the nonperturbative answer, when expanded with a small coupling constant, reduced to the perturbative result but with the IR regulator replaced by the (dimensionful) coupling constant.

One motivation for the original studies was that in the high-temperature limit of four-dimensional Yang-Mills theory there is an effective dimensional reduction to three dimensions, and the dimensionally reduced theory is superrenormalizable (see, for example, [2-4]). Here our motivation comes from a new application of massless

Published by the American Physical Society under the terms of the Creative Commons Attribution 4.0 International license. Further distribution of this work must maintain attribution to the author(s) and the published article's title, journal citation, and DOI. Funded by SCOAP. superrenormalizable theories: such theories appear in holographic models for the very early Universe [5].

The models introduced in [5] are based on threedimensional $S U(N)$ Yang-Mills theory coupled to massless scalars $\varphi$ in the adjoint of $S U(N)$ with a $\varphi^{4}$ interaction. To compute the predictions of these models for cosmological observables, one needs a nonperturbative evaluation of the relevant quantum field theory (QFT) correlators. This is the case even in the regime where the effective expansion parameter is small because of the IR singularities discussed above. Moreover, understanding the IR behavior of this QFT is important for another reason: in holographic cosmology cosmic evolution corresponds to inverse RG flow, and the initial singularity in the bulk is mapped to the IR behavior of the dual QFT. Thus, a mechanism for curing the IR singularities would also provide a holographic resolution of the initial bulk singularity.

In this Letter, we initiate the study of such a theory using lattice methods. We will focus on the simplest theory within this class: a three-dimensional massless scalar QFT with $\varphi$ in the adjoint of $S U(N)$ and a $\varphi^{4}$ interaction regularized on a Euclidean space-time lattice [6,7]. It turns out this theory still provides an interesting holographic model. Irrespective 
of the holographic motivation, we believe that understanding the fate of IR singularities in this QFT is an interesting problem in its own right, and this model provides the possibility to explicitly test the hypothesis in $[1,2]$.

We address two central questions in this Letter: Is the theory nonperturbatively IR finite, and what is the critical mass, i.e., what is the value of the bare mass such that the renormalized theory is massless? The latter question is crucial for future simulations at the massless limit where the holographic duality is defined [5]. Through two loops the critical mass is both linearly UV divergent and logarithmically IR divergent. We proceed to a nonperturbative determination of the critical mass in Markov Chain Monte Carlo simulations of the discretized Euclidean path integral, where naively the inverse of the finite extent of the lattice $L$ acts as the only IR regulator. By studying the finite-size scaling (FSS) nonperturbatively, within the effective theory and on the lattice, we find evidence for the absence of the IR divergence beyond perturbation theory.

The $N=2$ model is equivalent to the $O(3)$ vector model, and the $N=3$ model is in the same universality class as the $O(8)$ vector model [8]. These models have been studied widely in the literature [9], including studies of their critical mass and other values of $N$ [10-12]. For $N>3$, such an equivalence is not obvious, and little is known about the theories' phase structure (see, for example, [13]).

Lattice perturbation theory.-We consider the threedimensional Euclidean scalar $S U(N)$ valued $\varphi^{4}$ theory

$S=\int d^{3} x \operatorname{Tr}\left\{\left[\partial_{\mu} \varphi(x)\right]^{2}+\left(m^{2}-m_{c}^{2}\right) \varphi(x)^{2}+\lambda \varphi(x)^{4}\right\}$

with fields $\varphi=\varphi^{a}(x) T^{a}$, where $\varphi^{a}(x)$ is real and $T^{a}$ are the generators of $S U(N)\left(\operatorname{Tr}\left[T^{a} T^{b}\right]=\frac{1}{2} \delta_{a b}\right)$. In the following, we prefer to work with a rescaled version of the action where the 't Hooft scaling is explicit:

$S=\frac{N}{g} \int d^{3} x \operatorname{Tr}\left\{\left[\partial_{\mu} \phi(x)\right]^{2}+\left(m^{2}-m_{c}^{2}\right) \phi(x)^{2}+\phi(x)^{4}\right\}$,

which we obtain from Eq. (1) by identifying $\phi=\sqrt{N / g} \varphi$ and $\lambda=g / N$, where $g$ is the 't Hooft coupling, which should be kept fixed in the large $N$ limit. The field $\phi$ and coupling constant $g$ have mass dimension 1 .

To discretize the theory on a 3D space-time lattice with lattice spacing $a$, we replace partial derivatives by finite differences, $\partial_{\mu} \phi(x) \rightarrow \delta_{\mu} \phi(x)=[\phi(x+\hat{\mu} a)-\phi(x)] / a$, and integrals by sums $\int d^{3} x \rightarrow a^{3} \sum_{x \in \Lambda^{3}}$, where $a$ is the lattice constant, $\hat{\mu}$ a unit vector in the $\mu$ direction, and $\Lambda^{3}$ is the set of all lattice sites. We use periodic boundary conditions.

The diagrams that contribute to the critical mass $m_{c}^{2}$ at the two-loop level are shown in Fig. 1. The IR-finite but linearly UV-divergent one-loop integral is

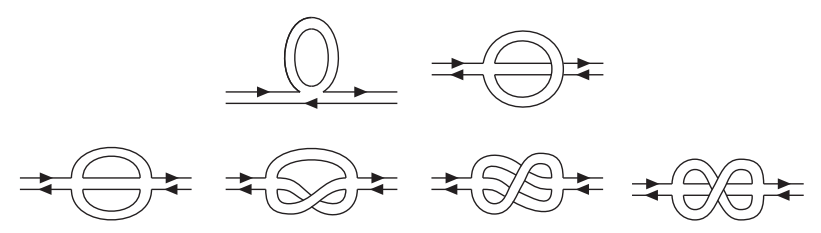

FIG. 1. One- and two-loop diagrams contributing to the mass renormalization in double-line representation representing matrix indices of the scalar propagator.

$$
\int_{-\pi / a}^{\pi / a} \frac{d^{3} k}{(2 \pi)^{3}} \frac{1}{\hat{k}^{2}}=\frac{Z_{0}}{a} \quad \text { with } \quad Z_{0}=0.252731 \ldots
$$

for lattice momenta $\hat{k}=(2 / a) \sin (k a / 2)$. The integral to be evaluated at two loops with vanishing external momentum $p=0$ is

$$
D(p)=\int_{-\pi / a}^{\pi / a} \frac{d^{3} k}{(2 \pi)^{3}} \frac{d^{3} q}{(2 \pi)^{3}} \frac{1}{\hat{k}^{2} \hat{q}^{2} \hat{r}^{2}}
$$

where $r=-k-q-p$, and hatted quantities are defined as above. By naive dimensional counting, confirmed by repeating the analysis of the IR properties of this diagram in [14] for $d=3$, we find that the integral diverges logarithmically in the IR as follows:

$$
D(p) \stackrel{p \rightarrow 0}{=} D_{\mathrm{IR}}(p)=-\frac{\log (|p a|)}{(4 \pi)^{2}}
$$

(see the derivation in Sec. I of the Supplemental Material [15]).

Following [1,2], we choose to introduce an IR regulator by setting the external momentum to $g /(4 \pi N) \equiv \Lambda$. (While the expression for the logarithmic cut-off dependence for a given UV regulator can be computed in perturbation theory $[16,17]$, the precise form for the IR regulator is unknown.) The two-loop expression for the critical mass then evaluates to

$$
m_{c}^{2}(g)=-g \frac{Z_{0}}{a}\left(2-\frac{3}{N^{2}}\right)+g^{2} D(\Lambda) \mathcal{N}(N),
$$

where $\mathcal{N}(N)=1-6 / N^{2}+18 / N^{4}$ (see also [17]). Representative values for $D(\Lambda)$ and $m_{c}^{2}(g)$ at one and two loops for $N=2$ are listed in Table I. (We evaluate the two-loop lattice momentum integral using the Markov Chain Monte Carlo integrations implemented in vEGAS [18]. The error estimates we provide together with the results are statistical only.) For the range of couplings presented in the table, the change from one to two loops corresponds to a relative change in the range $1 \%$ to $6 \%$. Note that the $n$ loop $(n>2)$ contribution to the critical mass is power-law IR divergent: $\sim g^{2}(g / \Lambda)^{n-2}$. If $\Lambda \sim g$, as in the mechanism of $[1,2]$, such terms are finite and 
TABLE I. Results for the two-loop integral $D(\Lambda)$ and the critical mass in lattice perturbation theory.

\begin{tabular}{lccc}
\hline \hline & & \multicolumn{2}{c}{$\left(a m_{c}\right)^{2}, N=2$} \\
\cline { 3 - 4 }$a g$ & $D(\Lambda)$ & 1 loop & 2 loop \\
\hline 0.1 & $0.05469(19)$ & -0.03159 & -0.03125 \\
0.2 & $0.04953(13)$ & -0.06318 & -0.06194 \\
0.3 & $0.04783(13)$ & -0.09477 & -0.09208 \\
0.5 & $0.045311(92)$ & -0.15796 & -0.15088 \\
0.6 & $0.044134(90)$ & -0.18955 & -0.17962 \\
\hline \hline
\end{tabular}

proportional to $g^{2}$. On the other hand, if the theory is truly IR divergent, such terms would dominate in the IR.

Finite-size scaling for $m_{c}^{2}$.-In this section, we provide details and results of our nonperturbative studies toward the determination of the critical mass. Our strategy is to compute the critical mass as a function of the IR cut-off given in terms of the inverse lattice size $1 / L$ by means of FSS. The observable we consider is the Binder cumulant

$$
B=1-\frac{N}{3} \frac{\left\langle\operatorname{Tr}\left[M^{4}\right]\right\rangle}{\left\langle\operatorname{Tr}\left[M^{2}\right]\right\rangle^{2}},
$$

where $M$ is the magnetization matrix defined below, and $\langle\cdot\rangle$ indicates the expectation value under the Euclidean path integral.

For each choice of simulation parameters, we determine the bare input mass $\bar{m}^{2}(\bar{B}, g, L)$ in the critical region for which the Binder cumulant takes some suitably chosen value $\bar{B}$. The Binder cumulant in a finite volume of extent $L$ in the critical scaling region is described by a scaling function $f$ :

$$
\bar{B}=f\left(\left[\bar{m}^{2}(\bar{B}, g, L)-m_{c}^{2}(g)\right] / g^{2} x^{1 / \nu}\right),
$$

where $x=g L$ and $\nu$ is the critical exponent. Expanding $f$ in the vicinity of the critical mass, we find the leading FSS behavior

$$
\bar{m}^{2}(\bar{B}, g, L)=m_{c}^{2}(g)+g^{2} x^{-1 / \nu} \frac{\bar{B}-f(0)}{f^{\prime}(0)} .
$$

FSS in the continuum effective theory.-Before analyzing and interpreting simulation data for the FSS of the critical mass, we can gain further analytical understanding of the critical behavior. To this end, we consider the effective field theory (EFT) of the zero mode of the field $\phi$, i.e., the magnetization

$$
M=\frac{1}{L^{3}} \int d^{3} x \phi(x),
$$

and fluctuations $\chi$ around it, i.e., $\phi=M+\chi$. In the vicinity of the critical point, the long-distance contributions described by $M$ dominate, motivating us to consider the leading-order effective action

$$
S_{\text {eff }}=\frac{L^{3} N}{g}\left\{\left(m^{2}-m_{c}^{2}\right) \operatorname{Tr}\left[M^{2}\right]+\operatorname{Tr}\left[M^{4}\right]\right\} .
$$

Following [19], we quantize the theory under the finitevolume path integral and find integral expressions for the Binder cumulant (for details see Sec II of the Supplemental Material). Expanding again in the vicinity of the critical point, we recover Eq. (9) and compute the leadingorder predictions $\left.\nu\right|_{N=2,4}=2 / 3,\left.f(0)\right|_{N=2} \approx 0.5431$, and $\left.f^{\prime}(0)\right|_{N=2} \approx-0.03586$, and $f(0)_{N=4} \approx 0.4459$ and $f^{\prime}(0)_{N=4} \approx-0.02707$, respectively.

Lattice simulation.-We implemented the model in the GRID library [20,21] with both the hybrid Monte Carlo [22] and a heat-bath overrelaxation algorithm [23-26]. We generated ensembles of $O(100 \mathrm{k})$ field configurations for $N=2$, 4, volumes with $L / a=8,16,32,48,64,96$, 128 , couplings $a g=0.1,0.2,0.3,0.5,0.6$, and a number of bare mass parameters in the vicinity of the perturbative prediction for $m_{c}^{2}(g)$ in Eq. (6). By using a wide range of couplings, a large range of lattice volumes was covered $(0.8 \leq x \leq 76.8)$ while keeping simulation costs acceptable. (The simulation data as well as the PYTHON analysis code have been made publicly available as $[27,28]$.)

Using multihistogram reweighting [29], we obtained a continuous representation of the Binder cumulant as a function of the bare scalar mass. Example results for $B(N, g, L)$ are shown in the top panel of Fig. 2, and the reweighting is illustrated in the bottom panel. The analysis was carried out under bootstrap resampling [30]. We determined the integrated autocorrelation time $\tau_{\text {int }}$ for $M^{2}, M^{4}$, and $\phi^{2}$ with the method of [31], with the largest values being $\mathrm{O}(100)$. All data was binned into bins of size $\max \left(50,4 \tau_{\text {int }}\right)$. The reweighting allows for a model-independent determination of $\bar{m}^{2}(\bar{B}, g, L)$ by means of an iterative solution. Example results for $\bar{m}^{2}(\bar{B}, g, L)$ are listed in Table II. We note the proximity of these finite-volume results to the two-loop infinite-volume prediction listed in Table I.

Finite-size scaling analysis.-We now turn to the fitting of $\bar{m}^{2}(\bar{B}, g, L)$. Guided by Eq. (9), we chose the fit ansatz

$$
\begin{aligned}
\bar{m}^{2}(\bar{B}, g, L)= & \left.m_{c}^{2}(g)\right|_{1-\text { loop }}+g^{2} \alpha \\
& +g^{2}\left[x^{-1 / \nu} \frac{\bar{B}-f_{0}}{f_{1}}+\beta D_{\mathrm{IR}}\left(\Lambda_{\mathrm{IR}}\right) \mathcal{N}(N)\right] .
\end{aligned}
$$

The first term is the one-loop expression for the critical mass, and it removes the linear UV divergence perturbatively [cf. Eq. (6)]. The coefficient $\alpha$ includes potential residual scheme dependence in the IR or UV regulator, e.g., normalization factors in the argument of $D_{\mathrm{IR}}$, as well as the contribution from higher loops when $\Lambda_{\mathrm{IR}} \sim g$. The second 

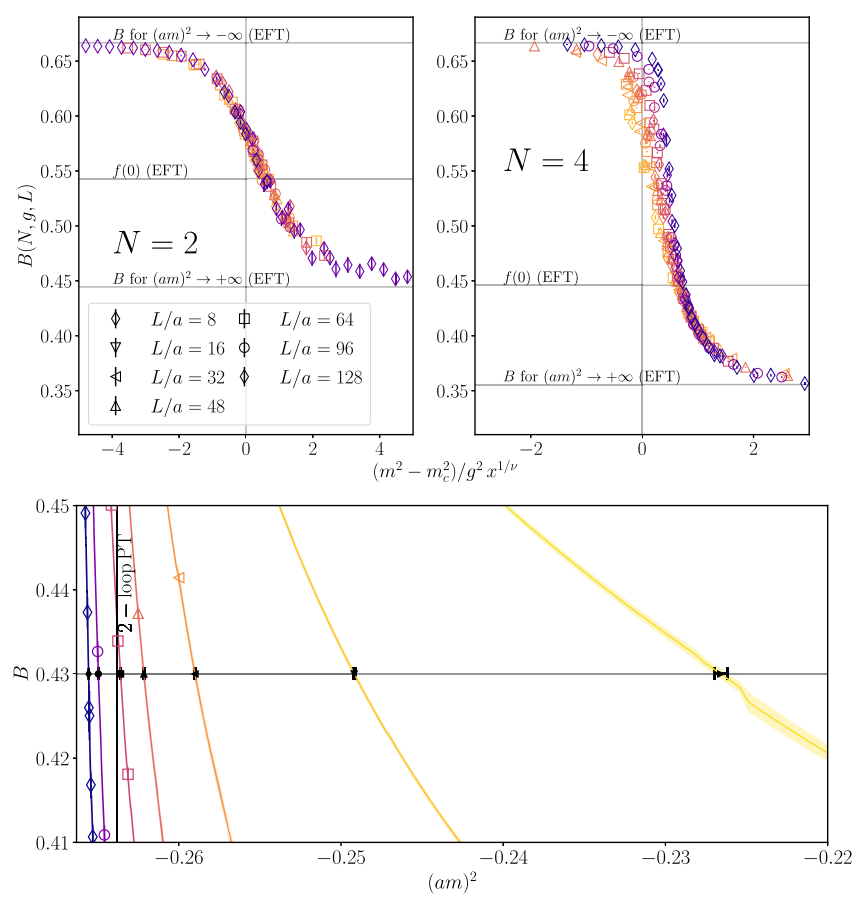

FIG. 2. Top: $N=2$ (left) and $N=4$ (right) results for the Binder cumulant, the EFT prediction for $f(0)$, and the value of the Binder cumulant in the limits $m^{2} \rightarrow \pm \infty$. The values on the $x$ axis have been rescaled using the values of the critical exponent $\nu$ and the critical masses $m_{c}^{2}$ determined in (cf. Table III). The darker colors correspond to larger values of $g L$. Bottom: Data points from simulations, lines from reweighting with width corresponding to the statistical error. Intersects of $N=4$, $g=0.6$ data for, from left to right, $L / a=128,96,64,48$, $32,8,16$ with $\bar{B}=0.43$ indicated with $y$-error bars. The black vertical line indicates the two-loop infinite-volume value of the critical mass.

term in brackets parameterizes the dependence on the IR cut-off for which we study, respectively, $\Lambda_{\mathrm{IR}}=$ $(1 / 4 \pi)(g / N)$ and $1 / L$. In the case $\Lambda_{\mathrm{IR}}=1 / L$, the $n$-loop IR-divergent contribution yields $g^{2} x^{n-2}$, which is of the same form as the finite scaling correction but with effective scaling dimension that tends to zero as $n \rightarrow \infty$. If such terms are present, their effects would dominate over the logarithmic or the finite size behavior in the IR. To better

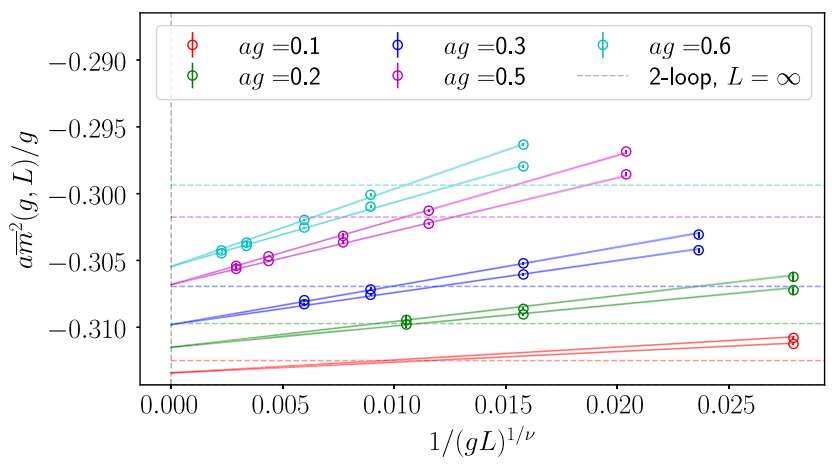

FIG. 3. Central fit $N=2, \bar{B}=0.52,0.53$. Dashed lines correspond to the two-loop prediction for the effective mass, solid lines to the fit result including error band. Value of $a g$ increasing from bottom to top. At each coupling, the top set of points corresponds to $\bar{B}=0.52$ and the bottom set to $\bar{B}=0.53$.

constrain the fit, we simultaneously analyze data from various pairings of two $\bar{B}$ values in the vicinity of $f(0)$ as predicted in the EFT. For $N=4$, we allowed one value of $\alpha$ per $\bar{B}$ value. For $N=2$, excellent fit quality was achieved without this additional freedom.

The central fits are for pairs $\bar{B}=\left.\{0.52,0.53\}\right|_{N=2}$ and $\left.\{0.42,0.43\}\right|_{N=4}$, respectively, for which we found the largest number of degrees of freedom described simultaneously. The ansatz in Eq. (12) provides an excellent parameterization ( $p$ values well above 5\%) for the simulation data over the entire range $g L_{\min } \gtrsim 12$ to $g L_{\max }=76.8$. The case $N=2$ is illustrated in Fig. 3 for $\Lambda_{\mathrm{IR}}=(1 / 4 \pi)(g / N)$. Table III summarizes the fit results. The first error is statistical, and, where applicable, the second error is the maximum shift of the fit result under variation of $g L_{\min }$ and the choice of $\bar{B}$ pairs with $\bar{B} \in$ $\left.\{0.51,0.52,0.53,0.54,0.55,0.56,0.57,0.58,0.59\}\right|_{N=2}$ and $\left.\bar{B} \in\{0.42,0.43,0.44,0.45,0.46,0.47\}\right|_{N=4}$, while requiring at least 15 d.o.f. Note that the result for $\beta$ is compatible with the prediction from perturbation theory, $\beta=1$ [cf. Eqs. (4) and (12)]. The result for $\nu$ for $N=2$ agrees well with a previous lattice determination [32], $\nu=$ $0.710(2)$. The EFT predictions for $\nu$ and $f(0)$ agree at the few-percent level. Fits with $\Lambda_{\mathrm{IR}} \propto 1 / L$ are not possible for similarly small values of $g L_{\min }$. For $N=2$, the first

TABLE II. Results for $(a \bar{m})^{2}(\bar{B}=0.53, g, L)$ for $N=2$.

\begin{tabular}{|c|c|c|c|c|c|}
\hline \multirow[b]{2}{*}{$L / a$} & \multicolumn{5}{|c|}{$a g$} \\
\hline & 0.1 & 0.2 & 0.3 & 0.5 & 0.6 \\
\hline 8 & $-0.024289(87)$ & $-0.05048(16)$ & $-0.07717(13)$ & $-0.12989(17)$ & $-0.15680(31)$ \\
\hline 16 & $-0.028398(37)$ & $-0.057413(65)$ & $-0.086163(97)$ & $-0.143556(77)$ & $-0.17205(13)$ \\
\hline 32 & $-0.030071(19)$ & $-0.060181(51)$ & $-0.090135(40)$ & $-0.149284(65)$ & $-0.178777(53)$ \\
\hline 48 & $-0.030595(21)$ & $-0.061032(37)$ & $-0.091267(47)$ & $-0.151126(48)$ & $-0.180582(51)$ \\
\hline 64 & $-0.030841(13)$ & $-0.061448(45)$ & $-0.091814(26)$ & $-0.151816(72)$ & $-0.181522(28)$ \\
\hline 96 & $-0.031067(12)$ & $-0.061811(16)$ & $-0.092270(31)$ & $-0.152521(29)$ & $-0.182345(66)$ \\
\hline 128 & $-0.0311266(93)$ & $-0.061962(43)$ & $-0.092486(29)$ & $-0.152808(33)$ & $-0.182680(29)$ \\
\hline
\end{tabular}


TABLE III. Results of $\chi^{2}$ fits to finite-size scaling data. The first error is statistical and the second systematic as described in the text.

\begin{tabular}{|c|c|c|c|c|c|c|c|c|c|c|}
\hline$N$ & $g L_{\min }$ & $g L_{\max }$ & $\alpha_{i}$ & $\nu$ & $\beta$ & $f(0)$ & $f^{\prime}(0)$ & $p$ & $\chi^{2} / N_{\text {d.o.f }}$. & $N_{\text {d.o.f }}$. \\
\hline 2 & 12.8 & 76.8 & $0.0019(8)(18)$ & $0.71(1)(6)$ & $1.05(5)(10)$ & $0.577(1)(16)$ & $-0.058(4)(53)$ & 0.2 & 1.2 & 31 \\
\hline 4 & 12.8 & 76.8 & $0.0010(5)(3) 0.0014(4)(4)$ & $0.840(8)(8)$ & $1.03(2)(2)$ & $0.497(1)(5)$ & $-0.090(3)(3)$ & 0.07 & 1.4 & 30 \\
\hline
\end{tabular}

acceptable $(p \geq 0.05)$ fit is possible only after discarding all data with $g L<32$ and for $N=4, g L<24$. The rhs axis in Fig. 4 shows how the $p$ value varies with the cut in $g L$. Generally, larger $p$ values for $\Lambda_{\mathrm{IR}} \propto g$ at a given value of $g L$ indicate that this ansatz provides a better description of the data in terms of a $\chi^{2}$ analysis. Inserting the fit parameters in Table III into Eq. (12) and taking the limit $x \rightarrow \infty$, we
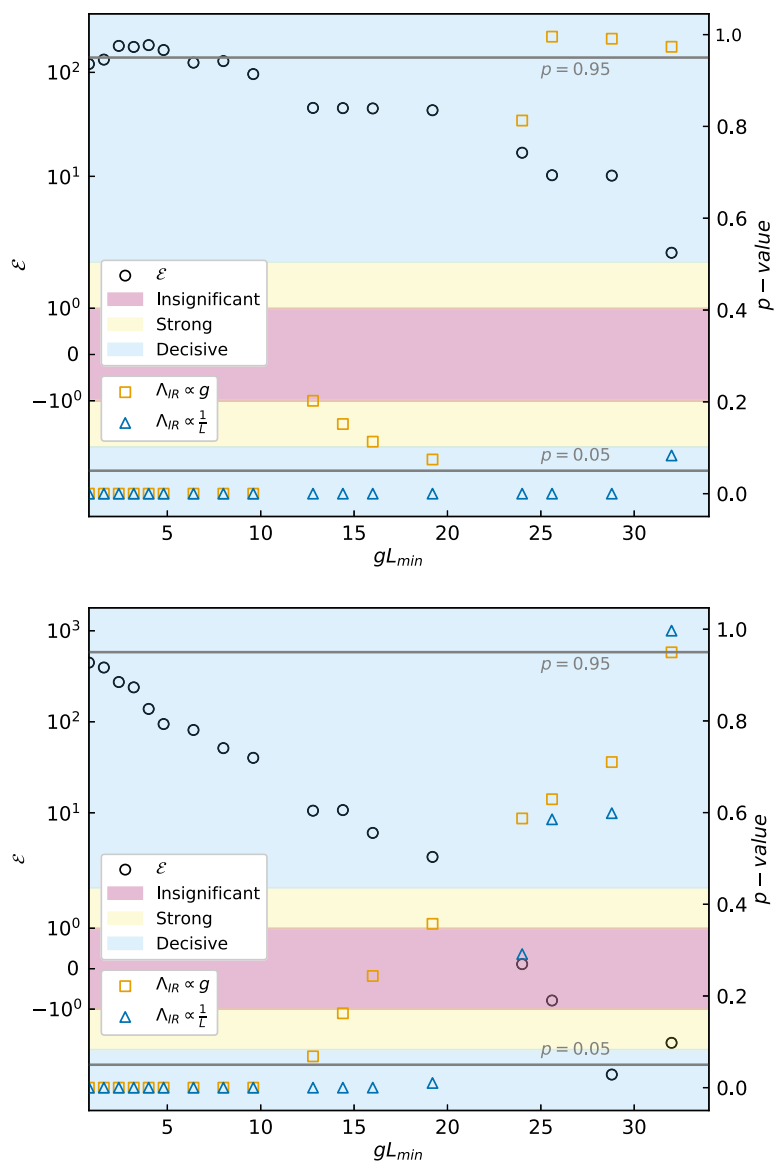

FIG. 4. Top: $N=2, \bar{B}=0.52, \bar{B}=0.53$ data. Bottom: $N=4$, $\bar{B}=0.42, \bar{B}=0.43$ data. The $p$ value of the fit of Eq. (12) with $\Lambda_{\mathrm{IR}} \propto g$ and $\Lambda_{\mathrm{IR}} \propto 1 / L$ (right $y$ axis) is shown by the orange squares and green triangles, respectively. The black circles represent the $\log$ in base 10 of the Bayes factor, $\mathcal{E}=\log _{10}(K)$, where $K=E_{1} / E_{2}$ with $E_{1}$ and $E_{2}$ being the marginal probabilities for fits with $\left(\Lambda_{\mathrm{IR}} \propto g\right)$ and $\left(\Lambda_{\mathrm{IR}} \propto 1 / L\right)$, respectively. The colored regions represent the strength of the evidence under the Jeffreys' scale [33]. Blue regions represent decisive evidence $(|\mathcal{E}|>2)$, while the yellow regions represent strong evidence $(1<|\mathcal{E}|<2)$ and the pink region represents insignificant evidence $(|\mathcal{E}|<1)$. obtain predictions for the infinite-volume critical mass. For instance, for $a g=0.1$ we find $\left(a m_{c}\right)^{2}=-0.031341(4)(6)$ for $N=2$ and $\left(a m_{c}\right)^{2}=-0.045515(2)(7)$ for $N=4$. If we assume the IR power divergences $D_{\mathrm{IR}}(x) \sim x^{n}(n=1$, $2,3,4)$ in lieu of logarithmic, no single acceptable fit is found $(p=0.00)$.

We also address the question of the IR regulator within the framework of Bayesian inference with uniform priors $\alpha \in[-0.4,0.4], f(0) \in[0,1], f^{\prime}(0) \in[-20,20]$, $\beta \in[-15,15]$, and $\nu \in[0,15]$. As in the frequentist study, for $N=4$, two values of $\alpha$ are used: $\alpha_{1,2} \in[-0.4,0.4]$. This analysis was done using PyMultiNest [34] as an interface to the MultiNest [35-37] code. The marginalized probabilities of each model $\left(\Lambda_{\mathrm{IR}} \propto g\right.$ and $\left.\Lambda_{\mathrm{IR}} \propto 1 / L\right)$ were calculated across a range of $g L_{\min }$ cuts and pairings of $\bar{B}$ values. In Fig. 4, both the $p$ value and the Bayes factor of the central fit are shown across the range of $g L_{\min }$ values. In this plot, the graph is broken down into regions according to the Jeffreys' scale [33]. The Bayes factor $K$ is $E_{1} / E_{2}$, where $E_{1}$ and $E_{2}$ are the marginal probabilities for model $1\left(\Lambda_{\mathrm{IR}} \propto g\right)$ and model $2\left(\Lambda_{\mathrm{IR}} \propto 1 / L\right)$, respectively. If $\mathcal{E}=\log _{10}(K)$ is greater than 1 , there is strong evidence for model 1 over model 2, and if it is greater than 2 , it is decisive. The reverse is true for negative values of $\mathcal{E}$ in support of model 2 . As the cut on $g L_{\min }$ is reduced (more data is used), the evidence for $\Lambda_{\mathrm{IR}} \propto g$ increases, with there being decisive evidence under the Jeffreys' scale for all $g L_{\text {min }}$ cuts for $N=2$ and for $g L_{\text {min }} \leq 19.2$ cuts for $N=4$. The same pattern is seen for all $\bar{B}$ values.

One can also obtain parameter estimates via the posterior probability distribution, which we find to be in excellent agreement with the results for the fit parameters from the $\chi^{2}$ analysis.

In conclusion, Bayesian inference prefers the IR-finite ansatz over the IR-divergent one; complementary and consistent with this, from $\chi^{2}$ fits we find the IR-finite FSS ansatz $\left(\Lambda_{\mathrm{IR}} \propto g\right)$ able to describe more degrees of freedom (i.e., a larger range in $g L$ ) with acceptable $p$ values.

Conclusions and outlook.-We present the first nonperturbative study of the critical properties of a three-dimensional superrenormalizable scalar QFT with $\varphi^{4}$ interaction and fields in the adjoint of $S U(N)$ with $N=2,4$. When studied in lattice perturbation theory, the theory exhibits a logarithmic IR divergence for the critical mass at the two-loop level. The absence of this divergence in our numerical results from lattice simulations provides strong evidence for the IR finiteness of the full theory. This constitutes one of the central results of this study. 
Further results are the nonperturbative determination of the critical masses. For the range of couplings considered here, the critical mass agrees with two-loop perturbation theory at and below the percent level when employing the dimensionful coupling constant $g$ as IR regulator, confirming the expectation of [1,2]. Our result for the critical exponent is close to the leading-order effective theory prediction, where the effective fields correspond to the magnetization of the full theory.

Three-dimensional superrenormalizable QFTs consisting of the Yang-Mills theory coupled to adjoint scalar and/ or fermionic matter are candidate theories for describing the physics of the early Universe by means of holographic duality. Our determination of the critical point constitutes the starting point for the study of cosmology from a threedimensional QFT. In view of the holographic duality, the cosmic evolution corresponds to inverse RG flow where the initial singularity is mapped to the IR behavior of the QFT. The absence of an IR singularity on the QFT side may thus be seen as the holographic resolution of the initial singularity in the bulk.

The authors would like to warmly thank Pavlos Vranas for his valuable support during the early stages of this project. We would like to thank Masanori Hanada for collaboration at early stages for this project. A. J. and K. S. acknowledge funding from STFC consolidated grants ST/ P000711/1 and ST/T000775/1. A. P. is supported in part by UK STFC grant ST/P000630/1. A. P., J. K. L. L., and H. B. $\mathrm{R}$ are funded in part by the European Research Council (ERC) under the European Unions Horizon 2020 research and innovation programme under Grant Agreement No. 757646 and A.P. additionally Grant Agreement No. 813942. J. K. L.L. is also partly funded by the Croucher Foundation through the Croucher Scholarships for Doctoral Study. B. K. M. was supported by the EPSRC Centre for Doctoral Training in Next Generation Computational Modelling Grant No. EP/L015382/1. L. D. D. is supported by an STFC Consolidated Grant, ST/ P0000630/1, and a Royal Society Wolfson Research Merit Award, WM140078. Simulations produced for this work were performed using the Grid Library ([38]), which is free software under GPLv2. This work was performed using the Cambridge Service for Data Driven Discovery (CSD3), part of which is operated by the University of Cambridge Research Computing on behalf of the STFC DiRAC HPC Facility ([39]). The DiRAC component of CSD3 was funded by BEIS capital funding via STFC capital grants ST/P002307/1 and ST/R002452/1 and STFC operations grant ST/R00689X/1. DiRAC is part of the National e-Infrastructure. The authors acknowledge the use of the IRIDIS High-Performance Computing Facility and associated support services at the University of Southampton in the completion of this work.
* Corresponding author. juettner@soton.ac.uk

[1] R. Jackiw and S. Templeton, How superrenormalizable interactions cure their infrared divergences, Phys. Rev. D 23, 2291 (1981).

[2] T. Appelquist and R. D. Pisarski, High-temperature YangMills theories and three-dimensional quantum chromodynamics, Phys. Rev. D 23, 2305 (1981).

[3] K. Farakos, K. Kajantie, K. Rummukainen, and M. E. Shaposhnikov, 3-D physics and the electroweak phase transition: Perturbation theory, Nucl. Phys. B425, 67 (1994).

[4] K. Kajantie, M. Laine, K. Rummukainen, and M. E. Shaposhnikov, The electroweak phase transition: A nonperturbative analysis, Nucl. Phys. B466, 189 (1996).

[5] P. McFadden and K. Skenderis, Holography for cosmology, Phys. Rev. D 81, 021301(R) (2010).

[6] J. K. L. Lee, L. Del Debbio, A. Jüttner, A. Portelli, and K. Skenderis, Towards a holographic description of cosmology: Renormalisation of the energy-momentum tensor of the dual QFT, in Proceedings of the 37th International Symposium on Lattice Field Theory (Lattice 2019) Wuhan, Hubei, China, 2019 (2019), arXiv:1909.13867.

[7] L. Del Debbio, E. Dobson, A. Jüttner, B. Kitching-Morley, J. K. Lee, V. Nourry, A. Portelli, H. B. Rocha, and K. Skenderis (LatCos Collaboration), Renormalization of the energy-momentum tensor in three-dimensional scalar $S U(N)$ theories using the Wilson flow, Phys. Rev. D 103, 114501 (2021). .

[8] F. Delfino, A. Pelissetto, and E. Vicari, Three-dimensional antiferromagnetic $\mathrm{CP}(N-1)$ models, Phys. Rev. E 91, 052109 (2015).

[9] M. Campostrini, M. Hasenbusch, A. Pelissetto, P. Rossi, and E. Vicari, Critical exponents and equation of state of the three-dimensional Heisenberg universality class, Phys. Rev. B 65, 144520 (2002).

[10] A. Pelissetto and E. Vicari, Critical mass renormalization in renormalized $\phi^{4}$ theories in two and three dimensions, Phys. Lett. B 751, 532 (2015).

[11] P. B. Arnold and G. D. Moore, Monte Carlo simulation of $O(2) \phi^{4}$ field theory in three-dimensions, Phys. Rev. E 64, 066113 (2001); Erratum, Phys. Rev. E 68, 049902 (2003).

[12] X.-p. Sun, Monte Carlo studies of three-dimensional $\mathrm{O}(1)$ and $O(4) \phi^{4}$ theory related to BEC phase transition temperatures, Phys. Rev. E 67, 066702 (2003).

[13] A. Pelissetto and E. Vicari, Three-dimensional ferromagnetic CP(N-1) models, Phys. Rev. E 100, 022122 (2019).

[14] M. Lüscher and P. Weisz, Coordinate space methods for the evaluation of Feynman diagrams in lattice field theories, Nucl. Phys. B445, 429 (1995).

[15] See Supplemental Material at http://link.aps.org/ supplemental/10.1103/PhysRevLett.126.221601 for "Nonperturbative infrared finiteness in superrenormalizable scalar quantum field theory".

[16] M. Laine, Exact relation of lattice and continuum parameters in three-dimensional SU(2) + Higgs theories, Nucl. Phys. B451, 484 (1995).

[17] M. Laine and A. Rajantie, Lattice continuum relations for 3D SU $(N)+$ Higgs theories, Nucl. Phys. B513, 471 (1998).

[18] P. Lepage, VEGAS, https://github.com/gplepage/vegas. 
[19] J. Zinn-Justin, Quantum Field Theory and Critical Phenomena, 4th ed., International Series of Monographs on Physics (Clarendon Press, Oxford, 2002).

[20] P. Boyle, A. Yamaguchi, G. Cossu, and A. Portelli, Grid: A next generation data parallel $\mathrm{C}++$ QCD library, arXiv: 1512.03487.

[21] P. A. Boyle, G. Cossu, A. Yamaguchi, and A. Portelli, Grid: A next generation data parallel $\mathrm{C}++$ QCD library, Proc. Sci., LATTICE2015 (2016) 023.

[22] S. Duane, A. D. Kennedy, B. J. Pendleton, and D. Roweth, Hybrid Monte Carlo, Phys. Lett. B 195, 216 (1987).

[23] F. R. Brown and T. J. Woch, Overrelaxed Heat-Bath and Metropolis Algorithms for Accelerating Pure Gauge Monte Carlo Calculations, Phys. Rev. Lett. 58, 2394 (1987).

[24] S. L. Adler, Overrelaxation algorithms for lattice field theories, Phys. Rev. D 37, 458 (1988).

[25] Z. Fodor and K. Jansen, Overrelaxation algorithm for coupled gauge-Higgs systems, Phys. Lett. B 331, 119 (1994).

[26] B. Bunk, Monte-Carlo methods and results for the electroweak phase transition, Nucl. Phys. B Proc. Suppl. 42, 566 (1995).

[27] B. Kitching-Morley and A. Jüttner, andreasjuettner/finitesize-scaling-analysis: V1.1—code release, https://doi.org/ 10.5281/zenodo.4290508 (2020).

[28] G. Cossu, H. Bergallo Rocha, L. Del Debbio, A. Jüttner, B. Kitching-Morley, J. K. Lee, A. Portelli, and K. Skenderis, Nonperturbative infrared finiteness in super- renormalisable scalar quantum field theory-data release, https://oi.org/ 10.5281/zenodo.4266114 (2020).
[29] A. M. Ferrenberg and R. H. Swendsen, New Monte Carlo Technique for Studying Phase Transitions, Phys. Rev. Lett. 61, 2635 (1988).

[30] B. Efron, Bootstrap methods: Another look at the Jackknife, Ann. Stat. 7, 1 (1979).

[31] U. Wolff (ALPHA Collaboration), Monte Carlo errors with less errors, Comput. Phys. Commun. 156, 143 (2004); Erratum, Comput. Phys. Commun. 176, 383 (2007).

[32] M. Hasenbusch, Eliminating leading corrections to scaling in the three-dimensional $O(N)$ symmetric $\phi^{4}$ model: $N=3$ and $N=4$, J. Phys. A 34, 8221 (2001).

[33] H. Jeffreys, The Theory of Probability (Oxford University Press, Oxford, 1998).

[34] J. Buchner, A. Georgakakis, K. Nandra, L. Hsu, C. Rangel, M. Brightman, A. Merloni, M. Salvato, J. Donley, and D. Kocevski, X-ray spectral modelling of the AGN obscuring region in the CDFS: Bayesian model selection and catalogue, Astron. Astrophys. 564, A125 (2014).

[35] F. Feroz and M. P. Hobson, Multimodal nested sampling: An efficient and robust alternative to markov chain Monte Carlo methods for astronomical data analyses, Mon. Not. R. Astron. Soc. 384, 449 (2008).

[36] F. Feroz, M. Hobson, and M. Bridges, MultiNest: An efficient and robust Bayesian inference tool for cosmology and particle physics, Mon. Not. R. Astron. Soc. 398, 1601 (2009).

[37] F. Feroz, M. P. Hobson, E. Cameron, and A. N. Pettitt, Importance nested sampling and the MultiNest algorithm, Open J. Astrophys. 2, 10 (2019).

[38] https://github.com/paboyle/Grid.

[39] https://www.dirac.ac.uk. 